\title{
Theoretical Investigation of Digital Predistortion System
}

\author{
Lou Jingyi $^{\text {a }}$, Qu Xiaoxu ${ }^{\text {b }}$ \\ ${ }^{1} 1$ College of Electronic Engineering, Naval University of Engineering, Wuhan 430033, China \\ ajingyi.lou@163.com, bConst.qq@163.com
}

Keywords: Power Amplifier, Predistortion, Indirect learning structure, Polynomial model.

\begin{abstract}
Some doubt has been cast upon the predistortion system applying indirect learning structure (IDLS). In this paper, using the polynomial model for the predistorter (PD), theoretical investigation and simulation on the predistortion system performance basing on IDLS is put out. The result shows that the performance of IDLS predistortion system is affected by PD modeling error and the measurement noise, and the upper bound of the system normalized nean square error (NMSE) caused by the measurement noise is just the noise to signal ration, and if the measurement noise is low enough, there is a NMSE floor caused by modeling error. This is a novel result which can help to make predict approximate estimation of the factual IDLS predistortion system performance.
\end{abstract}

\section{Introduction}

Power amplifier (PA) is indispensable for modern wireless communication systems, but the inherent nonlinearity of PA leads to signal distortion. Predistortion methods are widely investigated in recent years for compensating PA nonlinearity, and the baseband predistortion method is been focused on for its effectiveness and lower cost [1-3].

In exiting researches, there are two kinds of predistorter (PD) structure, direct learning structure (DLS) and indirect learning structure (IDLS). IDLS is put forward by Gao in 1991 to improve the linearity of speaker [4]. And in 2003 Marsalek applied IDLS in a polynomial modeled baseband predistortion system [5]. The key idea of IDLS is using postdistorter, which can distort the amplified signal back to its original form, as PD. Although it is proved by experiments that IDLS exhibits good in improving linearity of PA system [3, 6], there are still voices of doubt to it. For example, Morgan believed that noise inevitable found in measurement system bring bias error to the IDLS PD parameter estimation [7], which will degrade the PD performance significantly. As a result, later studies mainly concentrated on DLS Predistortion system [8], which has much complex algorithm and system structure.

In this paper, the effect of measurement noise and modeling error on IDLS PD algorithm is provided by theoretical analysis, and proved by simulation. This will be a direct evidence for the effectiveness of IDLS Predistortion system.

\section{System model}

Predistortion system using IDLS is shown in Fig. 1. The baseband equivalent model of PA is defined as $G(\bullet), u$ is the input signal and $v$ is the feedback signal of PA, defined as $v=G(u)$. The expected output of the postdistorter $F_{\text {post }}(\bullet)$ is $u$, i.e. $u=F_{\text {post }}(v)$. Obviously, $F_{\text {post }}(\bullet)$ is the inverse function of $G(\bullet)$, and $F_{\text {post }}(\bullet)=G^{-1}(\bullet)$. If PD is copied from postdistorter, it must satisfy $G\left(F_{p r e}(s)\right)=G\left(G^{-1}(s)\right)=s$. Here, PD is modeled as $L+1$ order polynomial:

$$
u=P D(v)=\sum_{l=0}^{L} c_{l} v|v|^{l}
$$


Where $c_{l}(l=0 . . L)$ is coefficient of the polynomial. The actual feedback signal is $y=v+a$, where $a$ is the measurement noise. In Morgan's view, because $E\left[|y|^{l}\right] \neq E\left[|v|^{l}\right]$, the estimation of $\hat{c}_{l}$ derived from $y$ is biased. But the amount of this bias is not given and the effect on system performance is not discussed.

In the following section, with measurement noise $a$, theoretical analysis and simulation on predistortion system using IDLS is shown, here Least-square (LS) algorithm is applied to estimate PD parameters $\hat{c}_{l}$.

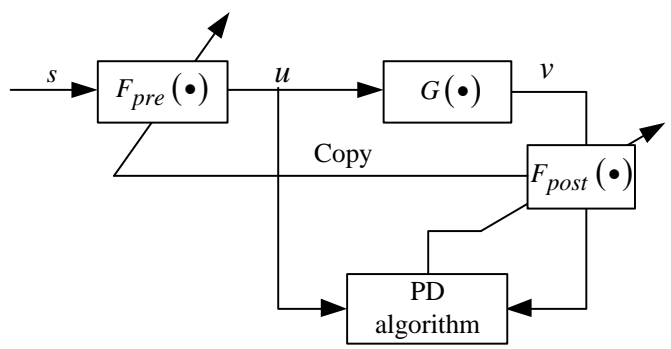

Fig. 1 Predistortion system using IDLS

\section{Performance analysis}

Theoretically, the order of the nonlinear PD polynomial, the inverse of nonlinear PA amplification characteristic function, is infinite. But in reality the computational complexity increases with the order of the polynomial, therefore the order is finite and modeling error is unavoidable. With considering the actual feedback signal $y=v+a$, PD can be expressed as

$$
u=\sum_{l=0}^{\infty} \hat{c}_{l} y|y|^{l}=\sum_{l=0}^{L} \hat{c}_{l}(v+a)|v+a|^{l}+\sum_{l=L+1}^{\infty} \hat{c}_{l}(v+a)|v+a|^{l}=\mathbf{v}^{T} \hat{\mathbf{c}}+\Delta \mathbf{v}^{T} \hat{\mathbf{c}}+\Delta u
$$

Where $\mathbf{v}=\left[\begin{array}{llll}v & v|v| & \cdots & v|v|^{L}\end{array}\right]^{T}$, and $\hat{\mathbf{c}}=\left[\hat{c}_{0}, \hat{c}_{1}, \cdots, \hat{c}_{L}\right]^{T}$ is the estimation value of PD parameters, and $\Delta u$ is the modeling error, and

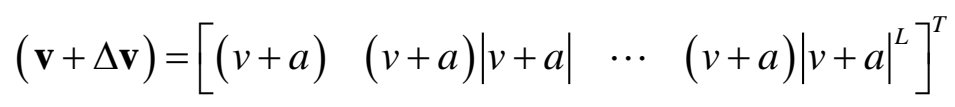

From (2) we can get the mean square error of the system with PD as follow

$$
M S E_{P D}=E\left\{\left(u-\mathbf{v}^{T} \hat{\mathbf{c}}\right)^{H}\left(u-\mathbf{v}^{T} \hat{\mathbf{c}}\right)\right\}=E\left\{\left(\Delta u+\Delta \mathbf{v}^{T} \hat{\mathbf{c}}\right)^{H}\left(\Delta u+\Delta \mathbf{v}^{T} \hat{\mathbf{c}}\right)\right\}
$$

In a general way, $E[u]=E[v]=0$, and $E[a]=0$, so from (1) we can get $E[\Delta u]=0$. Moreover, $\Delta u$ and $\Delta \mathbf{v}$ is uncorrelated, because they are caused by modeling error and measurement noise respectively. Then (3) can be simplified as

$$
M S E_{P D}=\sigma_{\Delta u}{ }^{2}+E\left[\hat{\mathbf{c}}^{H} \Delta \mathbf{v}^{*} \Delta \mathbf{v}^{T} \hat{\mathbf{c}}\right]
$$

According to (4), the error of IDLS predistortion system consists of two parts, $\sigma_{\Delta u}{ }^{2}$ caused by PD modeling error, is unavoidable for its polynomial model, and $E\left[\hat{\mathbf{c}}^{H} \Delta \mathbf{v}^{*} \Delta \mathbf{v}^{T} \hat{\mathbf{c}}\right]$, caused by measurement noise, is discussed as following.

Expand the amplitude of the summation of signal and noise as $|v+a|=\left[|v|^{2}+|a|^{2}+2|a||v| \cos \theta\right]^{1 / 2}$, and use Taylor series expanding the formula, we get 


$$
(v+a)|v+a|^{l}=v|v|^{l}+a|v|^{l}+\mathrm{O}(a), \quad l=0,1, \cdots L
$$

Neglect $\mathrm{O}(a)$, the high order term of $a$. From (2) and (5), we get $\Delta \mathbf{v}=a\left[\begin{array}{lll}1 & |v| & \cdots\end{array}|v|^{L}\right]^{T}$. Usually speaking, $|P A(v)| \geq|v|$, and $|P D(v)| \leq|v|$, therefore

$$
\mathbf{c}^{H} \Delta \mathbf{v}^{*} \Delta \mathbf{v}^{T} \mathbf{c}=\left.\left.\left|\sum_{l=0}^{L} c_{l}\right| v\right|^{l}\right|^{2}|a|^{2}=\left.|P D(v) / v|\right|^{2}|a|^{2} \leq|a|^{2}
$$

From (6) we can get

$$
E\left[\mathbf{c}^{H} \Delta \mathbf{v}^{H} \Delta \mathbf{v c}\right] \leq E\left[|a|^{2}\right]
$$

From (4) and (7), it can be concluded that polynomial modeled IDLS predistortion system suffers two kinds of error: one is the modeling error, which is determined by the polynomial model of PD; the other is the error causing by measurement noise, and the power of this part error is upper bounded by the measurement noise power itself.

\section{Simulations}

Linearization performance of predistortion system is simulated in this section, and we introduce the saleh model [9] as PA model. In the next simulation, the input signal $s$ is uniform distribution and the value range of $s$ is from -1 to 1, and system Normalized Mean Square Error (NMSE) is defined as following:

$$
\operatorname{NMSE}(d B)=10 \log _{10}\left[\sum_{n=0}^{N-1}|s-v|^{2} / \sum_{n=0}^{N-1}|s|^{2}\right]
$$

Fig. 2 is the relationship diagram between system NMSE and order of polynomial without measurement noise. In fig. 2, when L increases from 1 to 20, system NMSE drops from -22dB to around $-120 \mathrm{~dB}$. And when $\mathrm{L}$ is greater than 20, with the increasing of L, NMSE no longer drops. So we can come to the conclusion that when we use the polynomial model PD to compensate Saleh model PA, and once the order reaches a certain value, the modeling error do not reduce significantly, in other words, there is an inherent modeling error. Fortunately, the error is small enough and the effect on communication signal can be negligible.

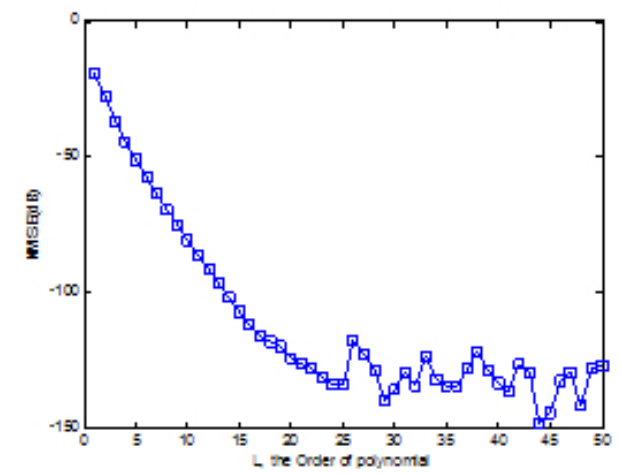

Fig.2. Relationship between system NMSE and polynomial order without measurement noise 


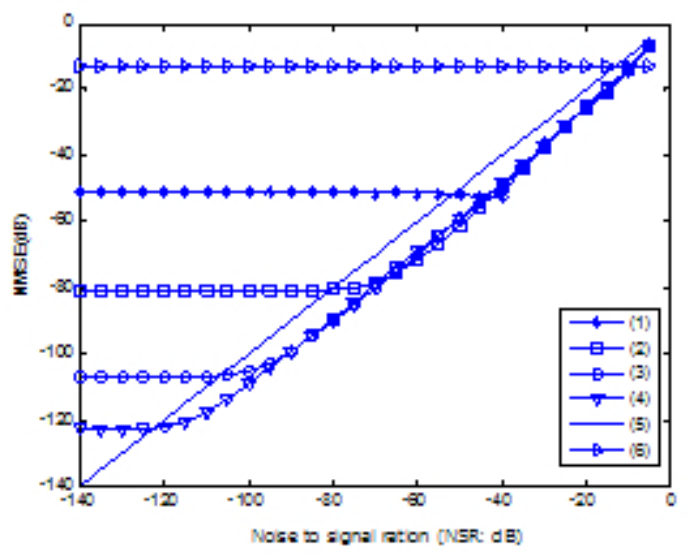

Fig. 3. Error performance of predistortion system using LS algorithm.

Fig. 3 is the diagram about error performance of predistortion system. The $\mathrm{x}$ label is the noise to signal ratio (NSR), defined as $E\left[|a|^{2}\right] / E\left[|s|^{2}\right]$. And the curve (1), (2), (3) and (4) is the system NMSE curve with polynomial order $L=5,10,15,20$, respectively. The curve (5) is NSR, the upper borderline of NMSE, and curve (6) is the value of $-12.7 \mathrm{~dB}$, which is the system NMSE with PA but without PD.

It is proved by Fig. 3 that the NMSE of predistortion system is determined by the order of the polynomial modeling PD and the measurement noise as the conclusion of the theoretical investigation. With the increasing of NSR, all the NMSE curves converge to the same value, which is the consequence of the dominance function of the measurement noise as compared to the modeling error. When the measurement noise is small, system performance is determined by the modeling error, therefore a NMSE floor is found in every curve.

In real system, there are two kinds of measurement noise in the feedback signal including background noise of PA and noise caused by the measurement circuit itself. The major noise is caused by analog-to-digital convertor. For example, the carrier to noise ratio of $12 \mathrm{bit} \mathrm{AD}$ converter AD9233, which is often used in high frequency predistortion experiment system, is about 70dBc. The noise of PA consist of channel thermal noise, shot noise and flicker noise, which often can be ignored compared with AD convertor noise. It means that using IDLS PD and LS algorithm, the system NMSE is just about $-70 \mathrm{~dB}$, but the value is $-12.7 \mathrm{~dB}$ without PD in the system for the nonlinearity of the PA. So we can get the conclusion that with the controllable measurement noise, the IDLS predistortion system is effective for compensating the nonlinearity characteristic of PA.

\section{Conclusions}

In this paper, performance of PD based on IDLS is evaluated through theoretical analysis and simulations. It is shown that system linearization performance of the predistortion system depends on both modeling error, which is determined by the polynomial model, and measurement noise. And it is proved theoretically that the upper bound of NMSE caused by measurement noise is the noise and signal ratio. This is a novel result and it may help people using IDLS PD techniques to make predict approximate estimation of the factual IDLS predistortion system performance, and then get correct trade-off between system implementation complexities and system performance.

\section{References}

[1] R. W. Santucci, M. K. Banava: Energy-Efficient Distributed Estimation by Utilizing a Nonlinear Amplifier, IEEE Transactions on Circuit and Systems, vol. 61(2014), p. 302-311.

[2] Q. Xiaoxu, L. Jingyi, G. Jun: Adaptive predistortion algorithm with optimum look-up table spacing, Journal of Huazhong University of science and technology, vol. 40(2012), p. 6-11. 
[3] M. Rawat, K. Rawat: Generalized Rational Functions for Reduced-Complexity Behavioral Modeling and Digital Predistortion of Broadband Wireless Transmitters, IEEE Transactions on Instrumentation and Measurement, vol. 63(2014), p. 485-498.

[4] F. X. Y. GAO, W. M. SNELGROVE: Adaptive linearization of a loudspeaker, International Conference on ICASSP, Verdun, Que., Canada(1991).

[5] R. Marsalek, P. Jardin, G. Baudoin: From post-distortion to pre-distortion for power amplifiers linearization, IEEE Communications Letters, vol. 7(2003), p. 308-310.

[6] H. Chun-hui, W. Yong-jie: Algorithm study of digital HPA Predistortion using one novel memory type BP neural network, Journal on Communications, vol. 35(2014), p. 16-23.

[7] M. D. R, M. Z. xiang: Reducing measurement noise effects in digital predistortion of RF power amplifiers, IEEE International Conference on Communications, Anchorage, Alaska, (2003).

[8] Y. Q. Qian: High-efficient adaptive predistortion structure for RF power amplifier linearization , Journal on Communication, Vol. 27(2006), p. 35-40.

[9] A. A. M. Saleh: Frequency-independent and frequency-dependent nonlinear models of TWT amplifiers, IEEE Transactions on Communications, Vol. 29(1981), p. 1715-1720. 